ESAIM: PROCEEDINGS AND SURVEYS, September 2018, Vol. 62, p. 1-16

Muhammad DAUHOO, Laurent DUMAS, Pierre GABRIEL and Pauline LAFITTE

\title{
MATHEMATICAL MODELING OF EFFECT OF MICROTUBULE-TARGETED AGENTS ON MICROTUBULE DYNAMIC INSTABILITY*
}

\author{
Ayuna Barlukova ${ }^{1}$, Stéphane Honoré $^{2}$ and Florence Hubert ${ }^{1}$
}

\begin{abstract}
Microtubule-targeted agents (MTAs), widely used in chemotherapy, are molecules that are able to block cancer cell migration and division. Their effect on microtubule (MT) dynamic instability is measured by their influence on observable parameters of MT dynamics such as growth speed, time-based catastrophe frequency, time-based rescue frequency, etc. In this paper, we propose a new mathematical model that is able to reproduce MT dynamics with an appropriate estimation of the main observable parameters. Using the experimental data on paclitaxel effect in presence of EB proteins, we fitted parameters of the model from several drug concentrations. It enable us to understand which non-observable model parameters are able to reproduce the effect of MTAs and thus to highlight a new potential mechanism of action associated with MTAs effect in presence of EB protein.
\end{abstract}

\section{INTRODUCTION}

Microtubule-targeted agents (MTAs) are currently widely used in chemotherapeutic regimens to treat different types of cancer including breast, lung, myelomas, lymphomas, and leukemias. They have a cytotoxic effect on dividing cells by affecting the $\mathrm{M}$ phase.

MTAs are divided into two groups, i.e. polymerizing and depolymerizing agents, depending on whether they increase or decrease MT polymer mass at high concentrations. At low concentrations both groups of MTAs impair MT functions and arrest cells in mitosis with little influence on polymerized mass [JW98]. It has been proposed that the common cytotoxic mechanism of all MTAs is kinetic stabilization (inhibition) of MT dynamics (growing and shortening) [JW98]. The suppression of MT dynamics by MTAs, such as paclitaxel, has been demonstrated both in vitro [DWJ95] and in cells [JW98]. More recently, the paradoxical effects of MTAs on MT dynamics in vitro, in presence of EB protein, has been revealed $\left[\mathrm{PHM}^{+} 12\right],\left[\mathrm{MKD}^{+} 13\right]$. Our aim is to better

* The program is funded thanks to the support of the AMIDEX PROJECT (No ANR-11-IDEX-0001-02) funded by the "INVESTISSEMENTS D'AVENIR" French Government program, managed by the French National Research Agency (ANR), and the support INSERM PLAN CANCER No PC201418.

${ }^{1}$ Aix Marseille Univ, CNRS, Centrale Marseille, I2M, Marseille, France

2 Aix-Marseille Univ, INSERM, CRO2, Marseille, France

(C) EDP Sciences, SMAI 2018 
understand this action of MTAs, such as paclitaxel, in different experimental conditions (i.e. drug concentration, presence/absence of EBs etc ...) using mathematical modeling. We want to study how the observable effect on "macroscopic" level can be produced by the MTA on the "microscopic" one. In particular, we reproduce the action of paclitaxel at various concentrations on MT dynamics in presence of EB protein through the model parameters.

We use the improved model based on the continuous model published by P. Hinow et al [HRT09]. In our previous work, we incorporated into the model [HRT09] a new variable $a$, called the "MT age" of the population of growing MTs. The value of the variable $a$ provides, in fact, information on the MT growth lifetime. It enables us to obtain the reliable estimations of average time- and distance-based catastrophe frequencies in our model $\left[\mathrm{BGH}^{+} 17\right]$.

In the present work, we use the same idea to evaluate the time-based rescue frequency. Indeed, we structure MTs in shortening state by the "shortening age" $a$. We use the information on the shrinkage lifetime to obtain the estimation on time-based rescue frequency. Thus, our new model of MT dynamics capture main measurable indicators frequently provided in literature: MT growth and depolymerization speeds, time-based catastrophe and rescue frequencies. This allows us to reproduce, through the model parameters, the MTA effect on MT dynamics described by a change in these four indicators for various drug concentrations.

\section{Continuous model of microtubule dynamics}

\subsection{Model}

The model is based on the continuous approach presented in [HRT09]. The time evolution of concentrations of free GTP- and GDP-tubulin are described by the functions of time $t, p(t)$ and $q(t)$, respectively. We denote the densities of the populations of growing and shrinking MTs by the functions $t \rightarrow u(t, \cdot, \cdot, \cdot)$ and $t \rightarrow v(t, \cdot, \cdot)$, respectively. We structure growing MTs by the length $x$, length of GTP-cap $z$ and MT age $a$. The variable $a$ has a dimension of time and starts from zero at the time a MT undergoes a transition from growth to shortening (catastrophe) or vice versa (rescue event). Growing MTs pass in shortening state when they loss their GTP caps, i.e. $z=0$. The shrinking MTs are structured by the length $x$ and the MT age $\tilde{a}$. Note that, thanks to this new variable, we can collect for each MT, the lifetime and the size variation of each of the growing or shortening events, allowing us to compute accurate indicators of the dynamic instabilites. This variable will also be use to reflect a possible aging phenomenon on the hydrolysis.

The dynamics of growing MTs is driven by GTP-tubulin addition rate (the growth rate) $\gamma_{p o l}(p(t)$ ) and by the rate of GTP hydrolysis $\gamma_{h y d r o}(a)$. The scheme of MT dynamics is presented on Fig. 1.1. The time evolution of MTs of this population is described by the following transport equation

$$
u_{t}+u_{a}+\operatorname{div}_{x z}(B(t, a) u)=0, \quad B(t, a)=\left(\begin{array}{c}
\gamma_{p o l}(p(t)) \\
\gamma_{p o l}(p(t))-\gamma_{h y d r o}(a)
\end{array}\right), a>0, x>z>0 .
$$

This model express the fact that the MT growth speed is a function $\gamma_{p o l}$ of the number of GTPtubulin with $\gamma_{p o l}$ an increasing function of GTP-tubulin concentration defined in the following way

$$
\gamma_{p o l}(p)=\alpha_{p o l} \times \mathbb{1}_{\left(p>p_{s}\right)}+\left(\alpha_{p o l} \frac{p(t)-p_{c}}{p_{s}-p_{c}}\right) \times \mathbb{1}_{\left(p_{c}<p<p_{s}\right)} .
$$

The parameter $p_{c}$ stands for the critical concentration of GTP-tubulin required for polymerization. We assume that the growth speed saturates at high GTP-tubulin concentrations. We denote by the 


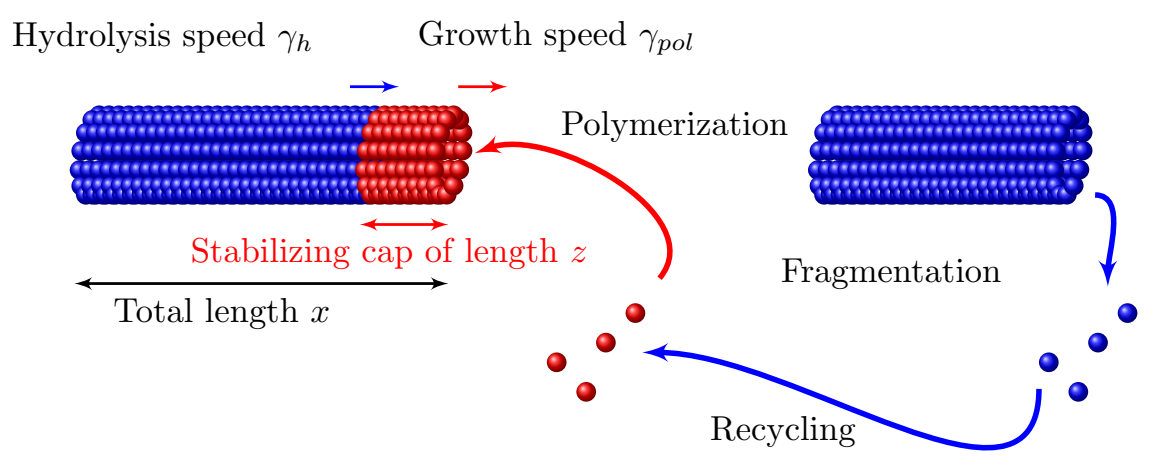

Figure 1. Main features of MT dynamics

parameter $p_{s}$ the saturating value of GTP-tubulin concentration and $\alpha_{p o l}$ is the maximal growth rate. If the quantity of GTP-tubulin $p(t)$ is between values $p_{c}$ and $p_{s}$, the growth rate depends linearly on $p(t)$. The transport equation (1) also says that the growth speed of the GTP-Tubulin cap is a balance between the polymerisation and hydrolysis velocities :

$$
R(t, a)=\gamma_{p o l}(p(t))-\gamma_{h y d r o}(a) .
$$

In our model, the GTP-hydrolysis mechanism is vectorial, which means that it propagates as a front along a MT body. The speed of the front dividing GTP- and GDP-tubulin dimers $\gamma_{\text {hydro }}(a)$, which we call GTP-hydrolysis rate is an increasing function of age $a$

$$
\gamma_{\text {hydro }}(a)=\gamma_{\text {hydro }}^{\text {young }} \times \mathbb{1}_{\left(a_{c}<a<a_{s}\right)}+\gamma_{\text {hydro }}^{\text {old }} \times \mathbb{1}_{\left(a_{s}<a\right)} .
$$

The parameter $a_{c}$ is a critical age that corresponds to the the time, observed in the experiment, necessary for the freshly incorporated GTP-tubulin dimer to be hydrolyzed. We set the GTPhydrolysis to be equal to zero for MT with an age less than $a_{c}$. For the MT with the age $a$ which is $a_{c}<a<a_{s}$, we set the GTP-hydrolysis rate to be equal to the parameter $\gamma_{\text {hydro }}^{\text {young }}$. We call these MTs "young ones". We call MTs with the age $a>a_{s}$ "old MTs" and set for them higher value of the GTP-hydrolysis rate $\gamma_{\text {hydro }}^{\text {old }}>\gamma_{\text {hydro }}^{\text {young }}$.

The nucleation process appears as a boundary condition on $\Gamma_{1}=\{a>0, x>0, z=x\}$

$$
\gamma_{\text {hydro }}(a) u(t, a, x, x)=\mathcal{N}(p) \psi(x) \Theta(a), \quad \mathcal{N}(p)=\mu p^{2}, t \in[0, \infty),
$$

where $\mu$ is a nucleation rate. We introduce here two non-negative functions $\Theta(a)$ and $\psi(x)$ such that

$$
\int_{0}^{\infty} \Theta(a) d a=1 \text { and } \int_{0}^{\infty} x \psi(x) d x=1
$$

with $\Theta$ and $\psi$ supported on supp $\Theta=\left(0, a_{0}\right)$, and supp $\psi=\left(0, x_{0}\right)$, respectively. The function $\psi(x)$ defines the normalized MT length distribution. 
Rescue events appear as a boundary condition on $\Gamma_{2}=\{a>0, x>0, z=0\}$

$$
R(t, a) u(t, a, x, 0)=\Theta(a) \lambda \int_{0}^{\infty} \mathbb{1}_{\left(\tilde{a}>a_{r e s}\right)} v(t, \tilde{a}, x) d \tilde{a}, \text { if } \mathbf{R}(\mathbf{t}, \mathbf{a})>\mathbf{0}, t \in[0, \infty) .
$$

Here, $\lambda$ characterizes the propensity for shrinking MTs to be rescued. The threshold $a_{r e s}$ is used to restrict the age $\tilde{a}$ of shrinking MTs that can return to the polymerization state.

We finally supply the system with a boundary condition on $\Gamma_{3}=\{(a, x, z)$ s.t. $a=0, x>0, z>0\}$ compatible with other boundary conditions

$$
u(t, 0, x, z)=0, \quad t \in[0, \infty) .
$$

The dynamics of the population of shrinking MTs is described by the transport equation

$$
v_{t}(t, \tilde{a}, x)+d i v_{a x}\left(B_{v} v\right)=I_{u \rightarrow v}-I_{v \rightarrow u}, \quad B_{v}=\left(\begin{array}{c}
1 \\
-\gamma_{\text {depol }}
\end{array}\right),
$$

endowed with

$$
v(t, 0, x)=0, t>0, x>0 .
$$

By $I_{u \rightarrow v}$ and $I_{v \rightarrow u}$ we denote influx and outflux to the domain of growing MTs that have the form

$$
I_{u \rightarrow v}=\boldsymbol{\Theta}(\tilde{\mathbf{a}}) \int_{0}^{\infty} R(t, a)^{-} u(t, a, x, 0) d a, \quad I_{v \rightarrow u}=\lambda \mathbb{1}_{\left(\tilde{a}>a_{r e s}\right)} v(y, \tilde{a}, x),
$$

The " - " operator is given in (10).

$$
x^{-}=\frac{1}{2}(-x+|x|) \text {. }
$$

The time evolution of GTP- and GDP-tubulin concentrations $p$ and $q$, respectvely, are described by following ordinary differential equations

$$
p_{t}=-\gamma_{p o l} I_{p \rightarrow u}+\kappa q-\mathcal{N}(p), \quad q_{t}=\gamma_{d e p o l} I_{v \rightarrow q}-\kappa q,
$$

where $I_{p \rightarrow u}=\int_{0}^{\infty} \int_{0}^{\infty} \int_{0}^{x} u(t, a, x, z) d z d x d a$ is the number of MTs in polymerization state, $\kappa$ is the rate of transformation of GDP-tubulin into GTP-tubulin, $I_{v \rightarrow q}=\int_{0}^{\infty} \int_{0}^{\infty} v(t, a, x) d x d a$ is the number of MTs in shrinkage state and $\gamma_{\text {depol }}$ is the constant depolymerization speed.

\subsection{Macroscopical output values of the model}

In the Table 1 you can find the notations of output macroscopical indicators of MT dynamics and their estimations. There are two groups of output values: observable and non-observable in the experiment. Note that in description of distance-based catastrophe frequency, we use the expression 


\begin{tabular}{|c|c|c|}
\hline Notation & Mathematical expression & Meaning \\
\hline \multicolumn{3}{|c|}{ Values observable in experiment } \\
\hline$\gamma_{\text {pol }}^{*}(t)$ & $\gamma_{p o l}(p(t))$ & $\begin{array}{c}\text { The growth (or polymerization) } \\
\text { speed (or rate) }\end{array}$ \\
\hline$F_{c a t}^{t e m p}(t)$ & $\frac{\int_{0}^{\infty} \int_{0}^{t} \frac{1}{a} \mathbb{1}_{(R(t, a)<0)} u(t, a, x, 0) d a d x}{\int_{0}^{\infty} \int_{0}^{t} \mathbb{1}_{(R(t, a)<0)} u(t, a, x, 0) d a d x}$ & Time-based catastrophe frequency \\
\hline$F_{\text {cat }}^{\text {spa }}(t)$ & $\frac{\int_{0}^{\infty} \int_{0}^{t} \frac{\mathbb{1}_{(R(t, a)<0)} u(t, a, x, 0) d a d x}{\int_{0}^{a} \gamma_{p o l}(t-a+s) d s}}{\int_{0}^{\infty} \int_{0}^{t} \mathbb{1}_{(R(t, a)<0)} u(t, a, x, 0) d a d x}$ & Distance(or space)-based catastrophe frequency \\
\hline$F_{\text {rescue }}^{\text {temp }}(t)$ & $\frac{\int_{0}^{\infty} \int_{0}^{t} \frac{1}{a} \mathbb{1}_{\left(a>a_{\text {res }}\right)} v(t, a, x) d a d x}{\int_{0}^{\infty} \int_{0}^{t} \mathbb{1}_{\left(a>a_{\text {res }}\right)} v(t, a, x) d a d x}$ & Time-based rescue frequency \\
\hline$F_{\text {rescue }}^{\text {spa }}(t)$ & $F_{\text {rescue }}^{\text {temp }}(t) / \gamma_{\text {depol }}$ & Distance-based rescue frequency \\
\hline$T_{\text {deco }}(t)$ & $L_{c a p}^{a v}(t) / \gamma_{p o l}(p(t))$ & Decoration time \\
\hline \multicolumn{3}{|c|}{ Values non-observable in experiment or hard to estimate } \\
\hline$I_{p \rightarrow u}(t)$ & $\int_{0}^{\infty} \int_{0}^{\infty} \int_{0}^{x} u(t, a, x, z) d z d x d a$ & The number of MTs in growth state \\
\hline$I_{v \rightarrow q}(t)$ & $\int_{0}^{\infty} \int_{0}^{\infty} v(t, a, x) d x d a$ & $\begin{array}{c}\text { The number of MTs in depolymerization } \\
\text { (or shrinking or shortening) state }\end{array}$ \\
\hline$L_{u}(t)$ & $\int_{0}^{\infty} \int_{0}^{\infty} \int_{0}^{\infty} x u(t, a, x, z) d z d x d a$ & Total tubulin in growing MTs \\
\hline$L_{v}(t)$ & $\int_{0}^{\infty} \int_{0}^{\infty} x v(t, a, x) d x d a$ & Total tubulin in shrinking MTs \\
\hline$L_{u}^{a v}(t)$ & $L_{u}(t) / I_{p \rightarrow u}(t)$ & Average length of MTs in growth state \\
\hline$L_{v}^{a v}(t)$ & $L_{v}(t) / I_{v \rightarrow q}(t)$ & $\begin{array}{c}\text { Average length of MTs in state of } \\
\text { depolymerization }\end{array}$ \\
\hline$L_{c a p}^{a v}(t)$ & $\frac{\int_{0}^{\infty} \int_{0}^{x} \int_{0}^{\infty} z u(t, a, x, z) d a d z d x}{I_{p \rightarrow u}(t)}$ & Average length of the GTP-cap \\
\hline$\gamma_{\text {hydro }}^{a v}(t)$ & $\frac{\int_{0}^{\infty} \int_{0}^{x} \int_{0}^{\infty} \gamma_{\text {hydro }}(a) u(t, a, x, z) d a d z d x}{I_{p \rightarrow u}(t)}$ & Average GTP-hydrolysis rate \\
\hline
\end{tabular}

TABLE 1. Estimations of indicators of MT dynamics in our model.

$\int_{0}^{a} \gamma_{p o l}(t-a+s) d s$ which is an increase in length of MT at time $t$ from time $t-a$ (time from rescue).

Decoration time $T_{\text {deco }}$ is the time of existence of EB1 binding sites $\left[\mathrm{PHM}^{+} 12\right]$. This formula of $T_{\text {deco }}$ is provided by the biological estimation. It is based on the assumption that the 
value of the growth rate, which is observable parameter, is probably close to the value of GTPhydrolysis rate which is non-observable. In our model, more appropriate alternative formula could be $L_{c a p}^{a v}(t) / \gamma_{h y d r o}^{a v}$. However, we choose the formula using as a biological estimation to be able to compare our results with experimental ones, in the future.

\section{Lemma 1 (Conservation of tubulin)}

The total quantity of tubulin in the system, which is a sum of free GTP- and GDP-tubulin, and the quantities of tubulin contained in MTs in both polymerization and depolymerization states, $\chi(t)=p(t)+q(t)+L_{v}(t)+L_{u}(t)$ is constant.

\subsection{Model parameters}

Our numerical results provided in [B17] showed that we can divide our parameters on two groups. In the first group are the parameters that mainly affect output "kinetic" indicators such as the growth speed, the time-based and distance-based catastrophe frequencies, the time-based and distance-based rescue frequencies without strong influence on proportion of polymerized-to-free tubulin mass. In another group are the parameters that poorly affect the "kinetic" indicators but strongly change the proportion of polymerized-to-free tubulin mass. In our simulation of paclitaxel effect we will use only the first group of parameters, since we will refer to the biological observations $\left[\mathrm{PHM}^{+} 12\right]$, describing the effect of MTAs at low concentrations, where few variation in proportion of polymer-to-free tubulin mass were observed. The description of the parameters of the first group is below.

\section{Parameters linked to depolymerization.}

- The parameter $\gamma_{\text {depol }}$ is directly given by the observable shortening rate.

- The parameter $a_{\text {res }}$ which is the time-delay before rescue event regulates the value of the time-based rescue frequency in our model. This time-based rescue frequency can also be adjusted by the parameter $\lambda$ which is the propensity of rescue. But we choose the parameter $a_{\text {res }}$ to regulate the time-based rescue frequency, since $a_{\text {res }}$ is simpler to interpret from the biological point of view than the parameter $\lambda$. Moreover, if we get the information on both shortening rate and the distance-based rescue frequency then we get an upper bound of $a_{\text {res }}$. In the case, when the distance based rescue frequency is not much affected by the drug the increase of the shortening rate probably lead to the decrease of the parameter $a_{\text {res }}$.

Thus, by the $\gamma_{\text {depol }}$ and $a_{\text {res }}$ we can fix the observable parameters shortening rate and time-based rescue frequency at the values that we get from the experiment.

Parameters linked to the GTP-hydrolysis. The parameters defining the GTP-tubulin hydrolysis rate can not be easily estimated in the experiment. These parameters are the initial time delay of the GTP-tubulin hydrolysis $a_{c}$, the two GTP-hydrolysis rates $\gamma_{\text {hydro }}^{\text {young }}$ and $\gamma_{\text {hydro }}^{\text {old }}$, and the threshold $a_{s}$ defining the age at which MTs undergo the acceleration of GTP-hydrolysis rate from $\gamma_{\text {hydro }}^{\text {young }}$ to $\gamma_{\text {hydro }}^{\text {old }}$. Our numerical results reveal that

- The parameter $a_{c}$ mainly affects the time-based catastrophe frequency and has a moderate effect on the growth speed.

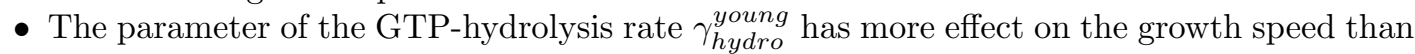
on time-based catastrophe frequency. 
- The parameter of the GTP-hydrolysis rate $\gamma_{\text {hydro }}^{\text {old }}$ has the similar effect as $\gamma_{\text {hydro }}^{\text {young }}$ on the growth speed and on time-based catastrophe frequency in the case $\left(\gamma_{\text {pol }}^{*}>\gamma_{\text {hydro }}^{\text {young }}\right)$. In the case $\left(\gamma_{\text {pol }}^{*}<\gamma_{\text {hydro }}^{\text {young }}\right)$ this parameter has poor or negligible effect on MT dynamics.

- In the case $\left(\gamma_{\text {pol }}^{*}>\gamma_{\text {hydro }}^{\text {young }}\right)$ the parameter $a_{s}$ has the similar effect on the growth speed and the time-based catastrophe frequency as the parameter $a_{c}$. Otherwise, this parameter has a weak or negligible effect on MT dynamics.

Both indicators $\gamma_{\text {pol }}^{*}$ and $F_{\text {cat }}^{\text {temp }}$ change in the same directions if we vary one of the four parameters $a_{c}, a_{s}, \gamma_{\text {hydro }}^{\text {young }}$ or $\gamma_{\text {hydro }}^{\text {old }}$. If, two parameters $a_{c}$ and $\gamma_{\text {hydro }}^{\text {young }}$ are sufficient to fit the growth rate and the time-based catastrophe frequency, we set $\gamma_{\text {hydro }}^{\text {old }}=\gamma_{\text {hydro }}^{\text {young }}, a_{s}=a_{c}$ that is equivalent to the one plateau of GTP-hydrolysis rate.

In presence of $\mathrm{EB}$ proteins, the experimental data provides information on $\mathrm{EB}$ comet length by fluorescence intensity of +end. As EB comet length is close to the GTP-tubulin cap length, therefore, one can estimate the decoration time. In our model, the parameter $a_{c}$ (of GTP-tubulin hydrolysis rate) correlates with this indicator. Thus, in our simulations we can take into account the data on EB fluorescence intensity adjusting the parameter $a_{c}$.

\section{NUMERICAL SCHEME FOR THE SYSTEM}

\subsection{Meshes}

- Let $0=t_{0}<\ldots<t_{n}<\ldots t_{N t}=T$ be a discretization of time interval $] 0, T$. The nonconstant time step, denoted by $d t_{n}=t_{n+1}-t_{n}$ is evaluate at each time step to ensure the stability of the scheme. Note that $d t_{n} \leq 0.001$ in the numerical results presented in this paper.

- Points of discretization in age will evaluate with each time step $d t_{n}$. On $n$-th iteration the points of discretization are $a_{k}^{n}=a_{k-1}^{n-1}+d t_{n-1}, k=1, \ldots, n$. Note that

$$
a_{k+1}^{n}-a_{k}^{n}=d t_{n-k}:=\varepsilon_{k}^{n}, \quad \text { with } \quad a_{0}^{n}=0
$$

and then $\varepsilon_{k+1}^{n+1}=\varepsilon_{k}^{n}, \forall k \geq 1$.

- We introduce a mesh $\mathcal{T}$ in $\mathbb{R}_{+}^{1}$ consisting of open intervals $\left.K_{i}=\right](i-1) h, i h\left[, i=1, \ldots, N_{x}\right.$ of the length $h$.

- Let $\mathcal{M}$ be a mesh of $\mathbb{R}_{+}^{2}$ of the domain $Z$ consisting of polygonal bounded convex subsets of $\mathbb{R}^{2}$. Notations are described in Figure 2 .

\subsection{Discrete equations}

The system (1)-(11) is discretized using an explicit Euler scheme in time, an upwind scheme for the transport terms and order one quadratures for the integral terms insuring the total preservation of tubulin. The time evolution of the quantity of free GTP-tubulin $p$ on a discrete level

$$
p^{n+1}=p^{n}-d t_{n}\left(\gamma_{p o l}\left(p^{n}\right) I_{p \rightarrow u}^{n}+\kappa q^{n}-\mathcal{N}(p)\right),
$$

where

$$
I_{p \rightarrow u}^{n}=\sum_{i=1}^{N_{x}} \sum_{j=1}^{i} h^{2} U_{i j}^{n}, \quad U_{i j}^{n}=\sum_{k=1}^{n} \varepsilon_{k}^{n} u_{k i j}^{n}
$$




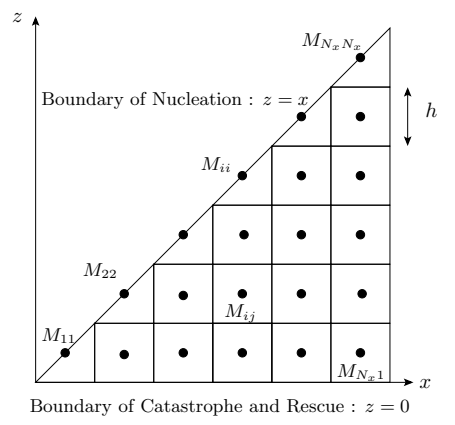

Figure 2. Mesh $\mathcal{M}$.

The time evolution of the quantity of free GDP-tubulin $q$ on a discrete level

$$
q^{n+1}=q^{n}+d t_{n}\left(\gamma_{\text {depol }} I_{v \rightarrow q}^{n}-\kappa q^{n}\right)
$$

where

$$
I_{v \rightarrow q}^{n}=h \sum_{k=1}^{n} \varepsilon_{k}^{n}\left(\sum_{i=1}^{N_{x}} h v_{k i}^{n}-\frac{h}{2} v_{k 1}^{n}\right) .
$$

The discrete equation for $v^{n+1}$ has the form

$$
v_{k, i}^{n+1}=v_{k-1, i}^{n}+d t_{n}\left(\frac{\gamma_{d e p o l}}{h}\left(v_{k-1 i+1}^{n}-v_{k-1 i}^{n}\right)-\lambda v_{k-1 i}^{n}\left(a_{k-1}^{n}>a_{r e s}\right)+\Theta_{1}^{n} I_{u \rightarrow v}^{n, i}\right),
$$

where $\quad I_{u \rightarrow v}^{n, i}=\sum_{k=1}^{n} \varepsilon_{k}^{n}\left(R_{k}^{n}\right)^{-} u_{k i 1}^{n}$, endowed with $v_{1 i}^{n}=0, \forall i$, and $R_{k}^{n}=\gamma_{p o l}\left(p^{n}\right)-\gamma_{h y d r o}\left(a_{k}^{n}\right)$.

Discrete equations for $u^{n+1}$ for squares cells $M_{i j}, i=2, \ldots, N_{x}, \quad j=1, \ldots, i-1, \quad k=2, \ldots, n$ are given by

$$
\begin{aligned}
u_{k i j}^{n+1}=u_{k-1 i j}^{n}-\frac{d t_{n}}{h} \gamma_{p o l}\left(p^{n}\right)\left(u_{k-1 i j}^{n}-u_{k-1 i-1 j}^{n}\right)-\frac{d t_{n}}{h}\left(R_{k-1}^{n}\right)^{-}\left(u_{k-1 i j}^{n}-u_{k-1 i j+1}^{n}\right) \\
-\frac{d t_{n}}{h}\left(R_{k-1}^{n}\right)^{+}\left(u_{k-1 i j}^{n}-u_{k-1 i j-1}^{n}\right),
\end{aligned}
$$

and for triangular cells $M_{i i}, i=1, \ldots, N_{x}, k=2, \ldots, n$

$$
\begin{aligned}
& u_{k i i}^{n+1}=u_{k-1 i i}^{n}-\frac{2 d t_{n}}{h} \gamma_{p o l}\left(p^{n}\right) u_{k-1 i i}^{n}-\frac{2 d t_{n}}{h}\left(R_{k-1}^{n}\right)^{-} u_{k-1 i i}^{n} \\
& +\frac{2 d t_{n}}{h}\left(R_{k-1}^{n}\right)^{+} u_{k-1 i i-1}^{n}+\frac{2 d t_{n}}{h} \gamma_{h y d r o}\left(a_{k-1}^{n}\right) u_{k-1 i i+1}^{n},
\end{aligned}
$$

endowed by the following conditions for all $n, i=1, \ldots, N_{x}, j=1, \ldots, i, \quad k=2, \ldots, n$

$$
u_{1 i j}^{n}=0, \quad u_{k i 0}^{n}=0, \quad u_{k i i+1}^{n}=0,
$$


and the boundary conditions

$$
\begin{gathered}
\gamma_{\text {hydro }}\left(a_{1}^{n}\right) u_{1 i i+1}^{n}=\mathcal{N}(p) \psi\left(x_{i}\right) \Theta_{1}^{n}, \\
R_{1}^{n} u_{1 i 0}^{n}=\lambda \Theta_{1}^{n} \sum_{\tilde{k} /\left(a_{\tilde{k}}^{n}>a_{\text {res }}\right)}^{n} \varepsilon_{\tilde{k}}^{n} v_{\tilde{k} i}^{n}, \quad \text { for } \quad i=1 \ldots, N_{x} .
\end{gathered}
$$

The boundary condition (6) at the discrete level reads as follows

$$
R_{1}^{n} u_{1 i 0}^{n}=\lambda \Theta_{1}^{n} \sum_{\tilde{k} /\left(a_{\tilde{k}}^{n}>a_{r e s}\right)}^{n} \varepsilon_{\tilde{k}}^{n} v_{\tilde{k} i}^{n}, \quad \text { for } \quad i=1 \ldots, N_{x} .
$$

\section{Biological oBSERVATions}

Consider the data from $\left[\mathrm{PHM}^{+} 12\right]$ given in Table 2 for the low dose of paclitaxel $(1 \mathrm{nM}, 10 \mathrm{nM}$, $100 \mathrm{nM}$ ) on MT dynamics in vitro, obtained in condition of presence of $75 \mathrm{nM}$ EB3. We can see that, at all doses of paclitaxel, all observable parameters are changed significantly compared to the control observation. Note that the shortening rate has the wide range of variability. Paclitaxel increased MT dynamic instability by increasing both MT growth and shortening rates. MT growth rates are increased in concentration dependent manner. Paclitaxel increased time-based rescue and catastrophe frequencies, while the space-based catastrophe frequency was poorly affected. The experimental data $\left[\mathrm{PHM}^{+} 12\right]$ also contains the information on the fluorescence intensity (mean FI in the Table 2) of EB3-GFP comets. To simulate the drug effect we use the information given in Table 2, including the fluorescence intensity of EB-binding site.

\begin{tabular}{|l|l|l|l|l|l|l|}
\hline & Growth rate & $\begin{array}{l}\text { Shortening } \\
\text { rate }\end{array}$ & $\begin{array}{l}\text { Time-based } \\
\text { catastrophe freq }\end{array}$ & $\begin{array}{l}\text { Space-based } \\
\text { catastrophe freq }\end{array}$ & $\begin{array}{l}\text { Time-based } \\
\text { rescue freq }\end{array}$ & Mean FI \\
\hline SI units & {$\left[\mu \mathrm{m} \mathrm{min}^{-1}\right]$} & {$\left[\mu \mathrm{m} \mathrm{min}^{-1}\right]$} & {$\left[\mathrm{min}^{-1}\right]$} & $\left.\mathrm{mm}^{-1}\right]$ & {$\left[\mathrm{min}^{-1}\right]$} & $\mathrm{AU}$ \\
\hline control & $3.87 \pm 1.00$ & $19.09 \pm 16.03$ & $1.72 \pm 0.12$ & $0.47 \pm 0.03$ & $2.12 \pm 0.29$ & $79.2 \pm 12$ \\
\hline \hline $\mathbf{1 n M}$ & $4.13 \pm 1.47$ & $17.87 \pm 11.24$ & $2.10 \pm 0.17$ & $0.58 \pm 0.04$ & $3.79 \pm 0.49$ & $72.8 \pm 18.8$ \\
\hline change in \% & +7 & -6 & +22 & +23 & +78 & -10 \\
\hline \hline 10nM & $4.89 \pm 1.56$ & $21.30 \pm 15.85$ & $2.62 \pm 0.20$ & $0.58 \pm 0.045$ & $5.77 \pm 0.63$ & $68.5 \pm 11.2$ \\
\hline change in \% & +26 & +12 & +52 & +23 & +172 & -15 \\
\hline \hline 100nM & $5.99 \pm 1.54$ & $28.12 \pm 25.85$ & $2.96 \pm 0.18$ & $0.50 \pm 0.033$ & $4.57 \pm 0.50$ & $58.2 \pm 15.6$ \\
\hline change in \% & +55 & +47 & +72 & +6 & +116 & -28 \\
\hline
\end{tabular}

TABLE 2. Parameters of MT dynamic instability of the MT +end in vitro. Control observation and paclitaxel effect on the parameters in presence of EB-proteins.

Rhomb diagram representation. On Fig. 3 you can see the graph corresponding to the experimental data given in the Table 2. Such kind of a graph, that we call a "rhomb diagram", was firstly presented in $\left[\mathrm{LBD}^{+} 14\right]$. It illustrates the action of paclitaxel on four main indicators of MT dynamics: depolymerization rate, growth rate, time-based catastrophe and rescue frequencies. This is a convenient representation to analyze the data on four parameters obtained at different drug concentrations. The diagram allows us visualize the tendency of changing of key parameters, as well as to see if this changing is in correlation with the drug concentration and also to visualize a 
relative change of the parameter to the control observation. All parameter values are normalized to the values of the control observation. On the Fig. 3 the red rhomb having its' vertices at $(-1,0)$ (shortening rate), $(0,1)$ (time-based rescue frequency), $(1,0)$ (growth speed) and at $(0,-1)$ (timebased catastrophe frequency) corresponds to the control observation. Other rhombs reflect the experimental data obtained in presence of paclitaxel at $1 \mathrm{nM}, 10 \mathrm{nM}$ and $100 \mathrm{nM}$ concentrations. Further we will use the rhomb diagrams to represent numerical results of our simulations.

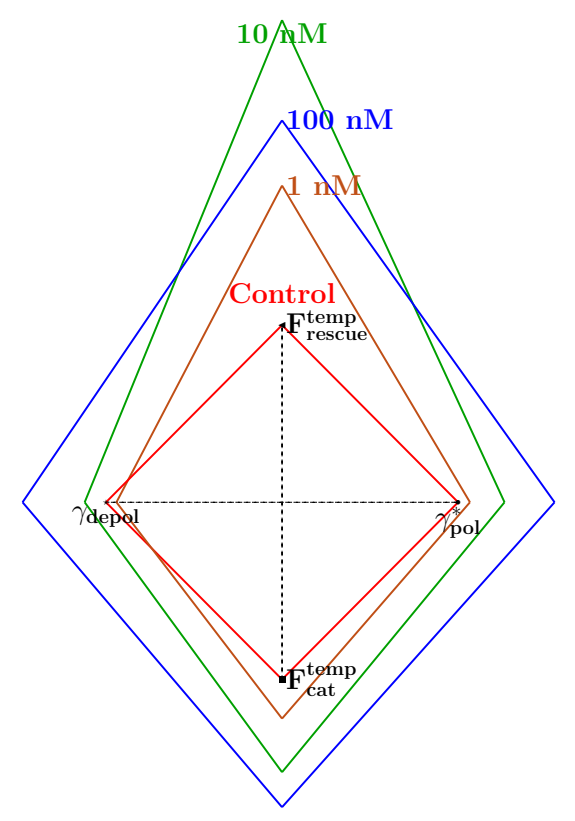

Figure 3. Rhomb diagram [LBD $\left.{ }^{+} 14\right]$. Paclitaxel effect at $1 \mathrm{nM}, 10 \mathrm{nM}$ and 100 $\mathrm{nM}$ in presence of $75 \mathrm{nM}$ of EB3 on four parameters: growth rate (right vertice), shrinking rate (left vertice), time-based catastrophe (below vertice) and rescue (above vertice) frequencies. All values are normalized to the values of the control observation.

\section{Strategy of Simulation of the PAClitaXel EFFECT}

The aim is to understand which of the model parameters can produce the output values close to ones obtained in the experiment at time $T$, when the system reaches the steady state.

The successive steps to calibrate the main parameters of the model for each drug concentration are the following:

- At first, we fix the parameters linked to depolymerization process.

(1) Fix $\gamma_{\text {depol }}$ according to the value of the shortening rate obtained in the experiment.

(2) Adjust the parameter $a_{r e s}$ to fit the time-based rescue frequency.

- Then fix the parameters of GTP-hydrolysis.

(3) reduce $a_{c}$ in agreement with the fluorescence intensity. This step is due to our observation that the parameter $a_{c}$ is found to regulate the decoration time which, in turn, correlates with fluorescence intensity. 
(4) Adjust $\gamma_{\text {hydro }}^{\text {young }}$ and if necessary involve the parameters of the second plateau of GTP hydrolysis rate: $a_{s}$ and $\gamma_{\text {hydro }}^{\text {old }}$ to fit $\gamma_{\text {pol }}^{*}$ and $F_{c a t}^{\text {temp }}$ with experimental growth speed and time-based catastrophe frequency. By default, $a_{s}=a_{c}$ and $\gamma_{\text {hydro }}^{\text {old }}=\gamma_{\text {hydro }}^{\text {young }}$ (i.e. only one plateau is considered), which corresponds to the absence of aging process.

This last step might be tricky and requires to perform a big number of numerical tests. This is due to the facts that all parameters $a_{c}, a_{s}, \gamma_{h y d r o}^{\text {young }}$ and $\gamma_{h y d r o}^{\text {old }}$, defining the function of GTPhydrolysis rate, regulate $\gamma_{p o l}^{*}$ and catastrophe frequencies. In particular, $\gamma_{p o l}^{*}$ depends on the average hydrolysis rate which, in turn, depends on interrelation of these four parameters.

Remark. Both $\gamma_{\text {hydro }}^{\text {young }}$ and $\gamma_{\text {hydro }}^{\text {old }}$ affect more the growth rate than catastrophe frequencies. In contrast, the time delay $a_{c}$ have stronger influence on time-based catastrophe frequency than on growth speed [B17].

\section{Results}

\subsection{Numerical results}

Fitting the model parameters. We started from the calibration of model parameters referring to the data corresponding to control observation given in Table 2. The obtained set of parameters can be found in Table 3. In all simulations, we switch off the nucleation process (i.e. we set $\mu=0$ ) at time $t=15 \mathrm{~min}$. The initial conditions are the following

$$
p(0)=15 \mu \mathrm{M}, \quad q(0)=0, \quad u(0, \cdot, \cdot, \cdot)=0, \quad v(0, \cdot, \cdot)=0 .
$$

The simulation performed with the set of parameters given in Table 3 we call "control test". It produces the values of growth speed, time- and distance-based catastrophe frequencies and of timebased rescue frequency which are in strong agreement with the experimental control observations given in Table 2 . The output values provided in Table 4 are obtained at time $T=20$ min, for which we already observe the steady state.

\begin{tabular}{|c|c|c|c|c|c|c|c|c|c|c|c|}
\hline \multicolumn{3}{|c|}{ Polymerization } & \multicolumn{4}{|c|}{ GTP-hydrolysis } & \multirow{2}{*}{$\begin{array}{c}\text { Depolymerization } \\
\gamma_{\text {depol }}\end{array}$} & \multicolumn{2}{|c|}{ Rescue } & Nucleation & GDP $\rightarrow$ GTP \\
\hline$p_{c}$ & $p_{s}$ & $\alpha_{p o l}$ & $a_{c}$ & $a_{s}$ & $\gamma_{\text {hydro }}^{\text {young }}$ & $\gamma_{\text {hydro }}^{\text {old }}$ & & $a_{\text {res }}$ & $\lambda$ & $\mu$ & $\kappa$ \\
\hline$\mu \mathrm{M}$ & $\mu \mathrm{M}$ & $\mu \mathrm{m} \min ^{-1} \mu \mathrm{M}^{-1}$ & $\mathrm{~s}$ & $\mathrm{~s}$ & $\mu \mathrm{m} \mathrm{min}^{-1}$ & $\mu m \min ^{-1}$ & $\mu \mathrm{m} \min ^{-1}$ & $\mathrm{~s}$ & $\min ^{-1}$ & $\mu \mathrm{M}^{-1} \min ^{-1}$ & $\min ^{-1}$ \\
\hline 2 & 15 & 32 & 3 & - & 4.3 & - & 19 & 26.7 & 19 & $5.9 \mathrm{e}-3$ & 2.4 \\
\hline
\end{tabular}

TABLE 3. Parameter set reproducing the MT dynamics in vitro of control observation described in $\left[\mathrm{PHM}^{+} 12\right]$

\begin{tabular}{|c|c|c|c|c|c|c|c|c|c|c|c|c|}
\hline \multicolumn{4}{|c|}{ Observable output indicators } & \multicolumn{4}{c|}{ Non-observable output indicators } \\
\hline $\boldsymbol{\gamma}_{\text {pol }}^{*}$ & $\boldsymbol{F}_{\boldsymbol{c a t}}^{\text {temp }}$ & $\boldsymbol{F}_{\boldsymbol{c a t}}^{\text {spa }}$ & $\boldsymbol{F}_{\boldsymbol{r e s c u e}}^{\text {temp }}$ & $\boldsymbol{T}_{\boldsymbol{d e c o}}$ & $\boldsymbol{\gamma}_{\boldsymbol{h y d r o}}^{\boldsymbol{a v}}$ & $\boldsymbol{L}_{\boldsymbol{u}}^{\boldsymbol{a v}}$ & $\boldsymbol{L}_{\boldsymbol{c a p}}^{\boldsymbol{a v}}$ & $\boldsymbol{L}_{\boldsymbol{v}}^{\boldsymbol{a v}}$ & $\boldsymbol{p}$ & $\boldsymbol{q}$ & $\boldsymbol{L}_{\boldsymbol{u}}$ & $\boldsymbol{L}_{\boldsymbol{v}}$ \\
\hline$\mu \mathrm{min}^{-1}$ & $\min ^{-1}$ & $\mu \mathrm{m}^{-1}$ & $\min ^{-1}$ & $\mathrm{~s}$ & $\mu m_{\mathrm{min}}^{-1}$ & $\mu \mathrm{m}$ & $\mu \mathrm{m}$ & $\mu \mathrm{m}$ & $\mu \mathrm{M}$ & $\mu \mathrm{M}$ & $\mu \mathrm{M}$ & $\mu \mathrm{M}$ \\
\hline $\mathbf{3 . 8 7}$ & $\mathbf{1 . 7 9}$ & $\mathbf{0 . 4 6}$ & $\mathbf{2 . 0 4}$ & $\mathbf{2 4 . 1 9}$ & 4.23 & 59.07 & 1.56 & 39.81 & 3.57 & 0.27 & 9.82 & 1.34 \\
\hline
\end{tabular}

TABLE 4. Output indicators of MT dynamics obtained for simulated "control observation" corresponding to the parameter set given in Table 3 .

Using our strategy we tried to simulate the effect of $1 \mathrm{nM}, 10 \mathrm{nM}$ and $100 \mathrm{nM}$ of the paclitaxel described in the Table 2. For this purpose, we change several parameters (including $\mu=0$ ) of the 
control set given in Table 3 at $t=15 \mathrm{~min}$. Thus, in all simulations we examine the influence of the parameter on the same MT population that is formed until $T=15 \mathrm{~min}$.

Numerical results obtained at each step of the strategy and the experimental data, as well, are further represented in rhomb diagrams for each drug concentration.

Simulation of $1 \mathrm{nM}$ paclitaxel effect. At the first step, we fix $\gamma_{\text {depol }}=17.9$ that has a negligible effect on other parameters (see Fig. 4(a)). After the second step, adjusting the parameter $a_{\text {res }}$, the time-based catastrophe frequency is strongly increased (see Fig. 4(b)). The change of this indicator appeared to be in the same direction as we want to obtain. However, the growth rate slightly reduced and changed in opposite direction to the one provided in the real data. We fix the value of $a_{c}$ at the value which is smaller than in the control test $(-10 \%)$ similar to the $10 \%$ decrease in fluorescence of $\mathrm{EB}$ comet $[\mathrm{PHM}+12]$. It results in the slight increase in time-based catastrophe frequency (see Fig. 4(c)). Then by performing a number of tests, we find the following set of parameters: $\gamma_{\text {depol }}=17.9, a_{\text {res }}=0.22, a_{c}=0.045, a_{s}=0.11, \gamma_{\text {hydro }}^{\text {young }}=3.5, \gamma_{\text {hydro }}^{\text {old }}=5$.

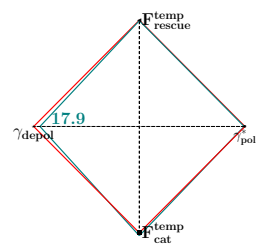

(a) $\gamma_{\text {depol }}=17.9$

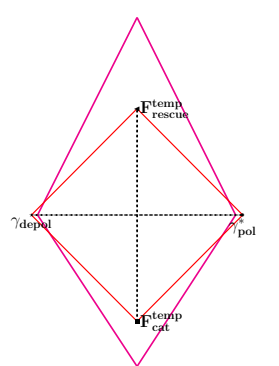

(b) $\gamma_{\text {depol }}=17.9$, $a_{\text {res }}=0.22$

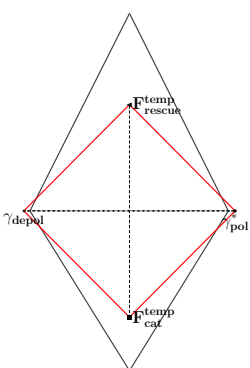

(c) $\gamma_{\text {depol }}=17.9,($ $a_{\text {res }}=0.22, a_{c}=0.045$

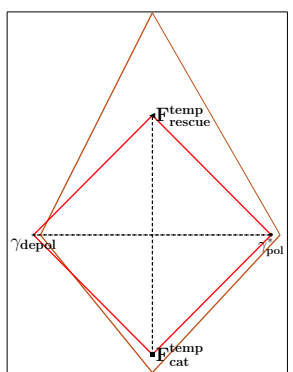

(d) Final simulation: $\gamma_{\text {depol }}=17.9, \quad a_{\text {res }}=$ $0.22, a_{c}=0.045, a_{s}=$ $0.11, \quad \gamma_{\text {hydro }}^{\text {young }}$ $3.5, \gamma_{\text {hydro }}^{\text {old }}=5$

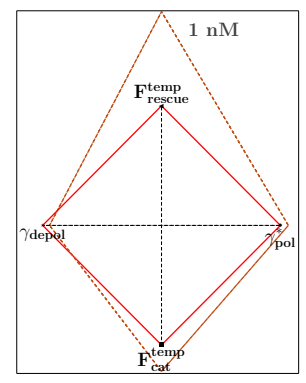

(e) Experimental data

Figure 4. Rhomb diagrams for observable indicators: the simulation of effect of $1 \mathbf{n M}$ paclitaxel.

\begin{tabular}{|c|c|c|c|c|c|c|c|c|c|c|c|c|}
\hline \multicolumn{4}{|c|}{ Observable output indicators } & \multicolumn{4}{c|}{ Non-observable output indicators } \\
\hline $\boldsymbol{\gamma}_{\text {pol }}^{*}$ & $\boldsymbol{F}_{\text {cat }}^{\text {temp }}$ & $\boldsymbol{F}_{\text {cat }}^{\text {spa }}$ & $\boldsymbol{F}_{\boldsymbol{r e s c u e}}^{\text {temp }}$ & $\boldsymbol{T}_{\boldsymbol{d e c o}}$ & $\boldsymbol{\gamma}_{\boldsymbol{h y d r o}}^{\boldsymbol{a v}}$ & $\boldsymbol{L}_{\boldsymbol{u}}^{\boldsymbol{a v}}$ & $\boldsymbol{L}_{\boldsymbol{c a p}}^{\boldsymbol{a v}}$ & $\boldsymbol{L}_{\boldsymbol{v}}^{\boldsymbol{a v}}$ & $\boldsymbol{p}$ & $\boldsymbol{q}$ & $\boldsymbol{L}_{\boldsymbol{u}}$ & $\boldsymbol{L}_{\boldsymbol{v}}$ \\
\hline$\mu \mathrm{min}^{-1}$ & $\mathrm{~min}^{-1}$ & $\mu \mathrm{m}^{-1}$ & $\mathrm{~min}^{-1}$ & $\mathrm{~s}$ & $\mu m \min ^{-1}$ & $\mu \mathrm{m}$ & $\mu \mathrm{m}$ & $\mu \mathrm{m}$ & $\mu \mathrm{M}$ & $\mu \mathrm{M}$ & $\mu \mathrm{M}$ & $\mu \mathrm{M}$ \\
\hline $\mathbf{4 . 1 6}$ & $\mathbf{2 . 0 6}$ & $\mathbf{0 . 5}$ & $\mathbf{3 . 7 9}$ & $\mathbf{1 3}$ & 4.74 & 54.83 & 0.93 & 45.47 & 3.69 & 0.3 & 9.21 & 1.8 \\
\hline
\end{tabular}

TABLE 5. Indicators of MT dynamics obtained for simulated effect of $\mathbf{1} \mathbf{n M}$ of paclitaxel.

Simulation of $10 \mathrm{nM}$ paclitaxel effect. After the first step, we observe a weak effect on timebased catastrophe frequency, which is reduced. After the second step, the catastrophe frequency changed in the direction corresponding to the drug effect, however a bit exceeded the required value. The growth speed moderately decreased and became too low compared with the experimental one which is strongly increased (see Fig. $5(\mathrm{~d})$ ). We continue to decrease $a_{c}$ in concentration dependent manner (see Fig. 5(c)) that does not influence on the parameters. The found set of parameters is $\gamma_{\text {depol }}=21.3, a_{\text {res }}=0.13, a_{c}=0.041, \gamma_{\text {hydro }}^{\text {old }}=6.3, a_{s}=0.1$. 


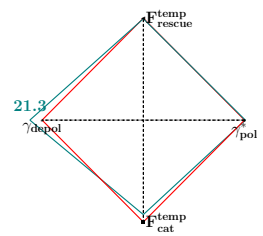

(a) $\gamma_{\text {depol }}=21.3$

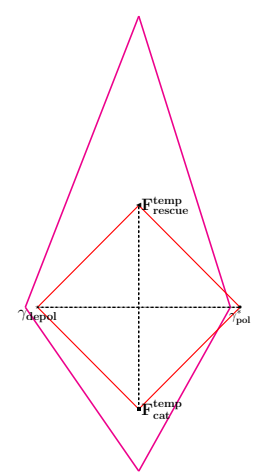

(b) $\gamma_{\text {depol }}=21.3$, $a_{\text {res }}=0.13$

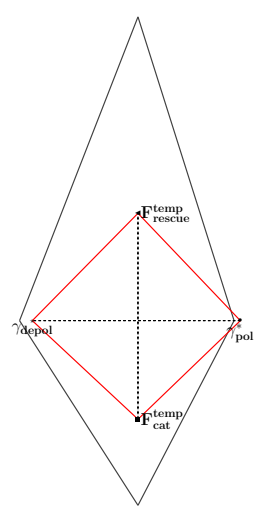

(c) $\gamma_{\text {depol }}=21.3$,
$a_{\text {res }}=0.13, a_{c}=0.041$

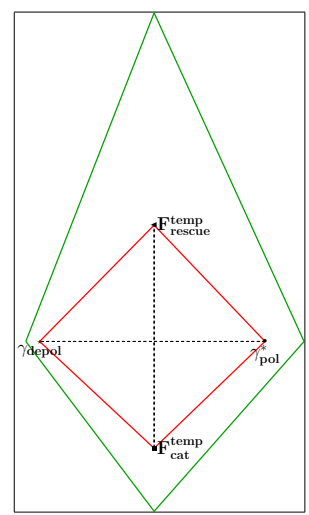

(d) Final simulation: $\gamma_{\text {depol }}=21.3, a_{\text {res }}=0.13$, $a_{c}=0.041, \gamma_{\text {hydro }}^{\text {old }}=6.3$, $a_{s}=0.1$

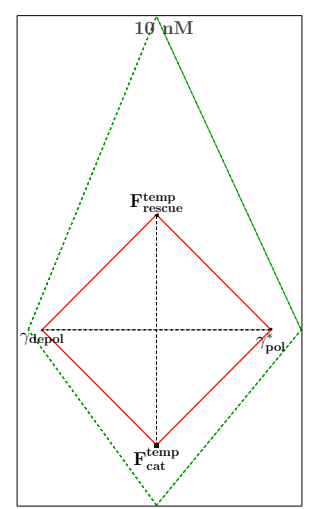

(e) Experimental data

Figure 5. The simulation of effect of $\mathbf{1 0} \mathbf{n M}$ paclitaxel.

\begin{tabular}{|c|c|c|c|c|c|c|c|c|c|c|c|c|}
\hline \multicolumn{4}{|c|}{ Observable output indicators } & \multicolumn{4}{c|}{ Non-observable output indicators } \\
\hline$\gamma_{\text {pol }}^{*}$ & $\boldsymbol{F}_{\text {cat }}^{\text {temp }}$ & $\boldsymbol{F}_{\text {cat }}^{\text {spa }}$ & $\boldsymbol{F}_{\boldsymbol{r e s c u e}}^{\text {temp }}$ & $\boldsymbol{T}_{\boldsymbol{d e c o}}$ & $\boldsymbol{\gamma}_{\boldsymbol{h y d r o}}^{\boldsymbol{a v}}$ & $\boldsymbol{L}_{\boldsymbol{u}}^{\boldsymbol{a v}}$ & $\boldsymbol{L}_{\boldsymbol{c a p}}^{\boldsymbol{a v}}$ & $\boldsymbol{L}_{\boldsymbol{v}}^{\boldsymbol{a v}}$ & $\boldsymbol{p}$ & $\boldsymbol{q}$ & $\boldsymbol{L}_{\boldsymbol{u}}$ & $\boldsymbol{L}_{\boldsymbol{v}}$ \\
\hline$\mu \mathrm{min}^{-1}$ & $\mathrm{~min}^{-1}$ & $\mu \mathrm{m}^{-1}$ & $\min ^{-1}$ & $\mathrm{~s}$ & $\mu m \min ^{-1}$ & $\mu \mathrm{m}$ & $\mu \mathrm{m}$ & $\mu \mathrm{m}$ & $\mu \mathrm{M}$ & $\mu \mathrm{M}$ & $\mu \mathrm{M}$ & $\mu \mathrm{M}$ \\
\hline $\mathbf{5 . 0 7}$ & $\mathbf{2 . 6 4}$ & $\mathbf{0 . 5 2}$ & $\mathbf{5 . 8 2}$ & $\mathbf{7}$ & 5.84 & 52.96 & 0.62 & 48.21 & 4.06 & 0.35 & 8.68 & 1.91 \\
\hline
\end{tabular}

TABLE 6. Indicators of MT dynamics obtained for simulated effect of $\mathbf{1 0} \mathbf{n M}$ of paclitaxel.

Simulation of $100 \mathrm{nM}$ paclitaxel effect. After the first step, we observe a strong influence on time-based catastrophe frequency, which is reduced and weak effect on the growth speed which is increased. After the second step of the strategy, we see the opposite effect on these two parameters. The growth speed is slightly reduced and the time-based catastrophe frequency is strongly increased compared to the control one. On the following step, we continue to decrease the parameter $a_{c}$ (see Fig. 6(c)) that increased the time-based catastrophe frequency and do not changed the growth speed. On the fourth steps, we increase $\gamma_{h y d r o}^{\text {old }}$ and strongly reduce $a_{s}$. The found set of parameters is $\gamma_{\text {depol }}=28, a_{\text {res }}=0.18, a_{c}=0.036, a_{s}=0.04, \gamma_{\text {hydro }}^{\text {old }}=7$.

\begin{tabular}{|c|c|c|c|c|c|c|c|c|c|c|c|c|}
\hline \multicolumn{4}{|c|}{ Observable output indicators } & \multicolumn{4}{c|}{ Non-observable output indicators } \\
\hline$\gamma_{\text {pol }}^{*}$ & $\boldsymbol{F}_{\text {cat }}^{\text {temp }}$ & $\boldsymbol{F}_{\boldsymbol{c a t}}^{\text {spa }}$ & $\boldsymbol{F}_{\boldsymbol{r e s c u e}}^{\text {temp }}$ & $\boldsymbol{T}_{\boldsymbol{d e c o}}$ & $\boldsymbol{\gamma}_{\boldsymbol{h y d r o}}^{\boldsymbol{a v}}$ & $\boldsymbol{L}_{\boldsymbol{u}}^{\boldsymbol{a v}}$ & $\boldsymbol{L}_{\boldsymbol{c a p}}^{\boldsymbol{a v} \boldsymbol{v}}$ & $\boldsymbol{L}_{\boldsymbol{v}}^{\boldsymbol{a v}}$ & $\boldsymbol{p}$ & $\boldsymbol{q}$ & $\boldsymbol{L}_{\boldsymbol{u}}$ & $\boldsymbol{L}_{\boldsymbol{v}}$ \\
\hline$\mu \mathrm{min}^{-1}$ & $\mathrm{~min}^{-1}$ & $\mu \mathrm{m}^{-1}$ & $\mathrm{~min}^{-1}$ & $\mathrm{~s}$ & $\mu m \min ^{-1}$ & $\mu \mathrm{m}$ & $\mu \mathrm{m}$ & $\mu \mathrm{m}$ & $\mu \mathrm{M}$ & $\mu \mathrm{M}$ & $\mu \mathrm{M}$ & $\mu \mathrm{M}$ \\
\hline $\mathbf{6 . 1 7}$ & $\mathbf{2 . 9 8}$ & $\mathbf{0 . 4 8}$ & $\mathbf{4 . 4 9}$ & $\mathbf{8}$ & 6.78 & 56.62 & 0.82 & 46.64 & 4.51 & 0.39 & 8.55 & 1.55 \\
\hline
\end{tabular}

TABLE 7. Indicators of MT dynamics obtained for simulated effect of $\mathbf{1 0 0} \mathbf{n M}$ of paclitaxel.

The comparison of the simulated effect for $1 \mathrm{nM}, 10 \mathrm{nM}$ and $100 \mathrm{nM}$ and experimental data are given on Fig. 7. The parameter values that were changed from the "control" are given in Table 8 for final simulations of the drug effect. 


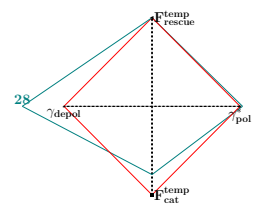

(a) $\gamma_{\text {depol }}=28$

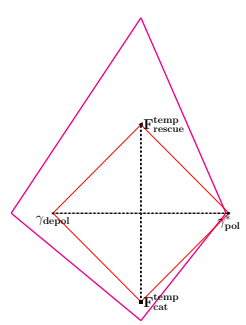

(b) $\gamma_{\text {depol }}$
$a_{\text {res }}=0.18$
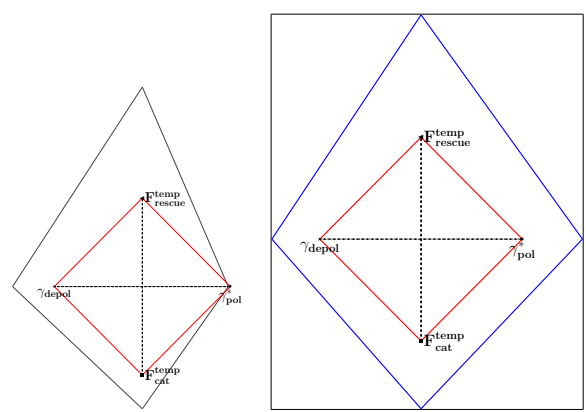

$28,(\mathrm{c}) \gamma_{\text {depol }}=28$,

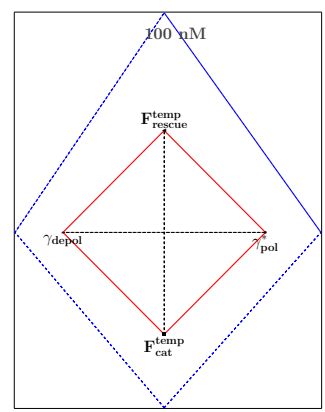

(e) Experimental data:

Figure 6. The simulation of effect of $\mathbf{1 0 0} \mathbf{n M}$ paclitaxel.

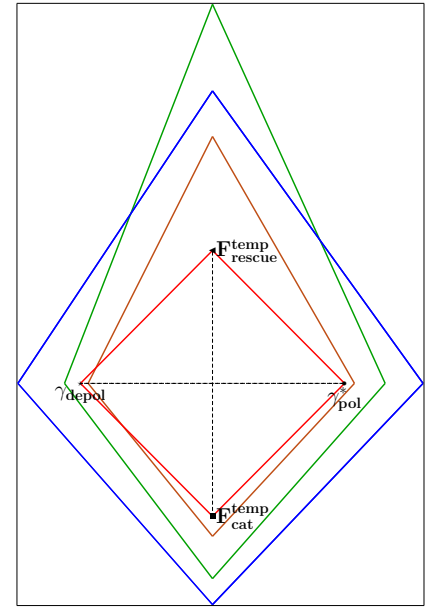

(a) Simulated effect

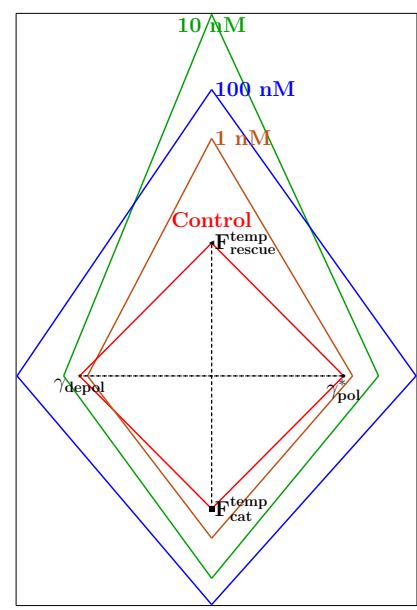

(b) Experimental data 1-100 $\mathrm{nM}$ $+\mathrm{EBs}$

Figure 7. Comparison of the simulated effect of $1 \mathrm{nM}, 10 \mathrm{nM}, 100 \mathrm{nM}$ of paclitaxel in presence of $\mathrm{EB}$ proteins with biological observations

\section{Conclusions AND Discussions}

We created a new model of MT dynamics allowing to simulate the MTA effect on four measurable indicators of dynamics: a growth rate, a depolymerization rate, a time-based catastrophe frequency and a time-based rescue frequency. We have tested our model by reproducing the paclitaxel effect in presence of EBs. 


\begin{tabular}{|l|l|l|l|l|}
\hline & Control & 1 nM & 10 nM & 100 nM \\
\hline \multicolumn{5}{|c|}{ Parameters "observable" in experiment } \\
\hline$\gamma_{\text {depol }}$ & 19 & 17.9 & 21.3 & 28 \\
\hline$a_{\text {res }}$ & 0.445 & 0.22 & 0.13 & 0.18 \\
\hline \multicolumn{4}{|c|}{ Parameters non-observable in experiment } \\
\hline$a_{c}$ & 0.05 & 0.045 & 0.041 & 0.036 \\
\hline$\gamma_{\text {hydro }}^{\text {young }}$ & 4.3 & 3.5 & 4.3 & 4.3 \\
\hline \multicolumn{4}{|c|}{ Parameters linked to aging effect in our model } \\
\hline$a_{\text {s }}$ & - & 0.11 & 0.1 & 0.04 \\
\hline$\gamma_{\text {hydro }}^{\text {old }}$ & - & 5 & 6.3 & 7 \\
\hline
\end{tabular}

TABLE 8. Set of main parameters of the model corresponding to the control and the simulated effect of paclitaxel in presence of EB3 protein.

Our model proposes a new concept of the GTP-hydrolysis that is vectorial, age-dependent and delayed. Our analysis showed that the parameter $a_{c}$ as well as parameters linked to MT aging can play a crucial role in regulation of the MT dynamics.

According to the model, we can determine the effect of paclitaxel on non-observable parameters in experiment. We find that in order to fully explain experimental results in presence of EBs paclitaxel might have an effect on the initial time delay of GTP-hydrolysis $a_{c}$ and increase the aging properties of a MT described by parameters $a_{s}$ and $\gamma_{\text {hydro }}^{\text {old }}$ in our model. The parameter $a_{s}$ (the time at which the GTP-hydrolysis accelerates) decreases and the second plateau of GTPhydrolysis $\gamma_{\text {hydro }}^{\text {old }}$ increases for higher concentrations of paclitaxel in presence of EB proteins.

Our results highlight a new potential mechanism of action associated with MTAs effect in presence of EB protein which is induction of MT aging by the GTP hydrolysis during MT growth, including the effect of the initial delay $a_{c}$. Such effect can be observed on kymographs in EB3-tip tracking assay. Further, we intend to test our strategies on simulation of destabilizing drug effects, such as Vinca Alcaloids.

One of the limits of our model is that it comprises the fixed shortening rate. The fluctuations in shortening rate among one population of MTs can be very strong. In further work, we can represent the depolymerization rate as a fragmentation of MTs to improve our model. We also do not consider in this work the pause state of MTs. It can be important in modeling of drug effects that are known to induce a pause state for a big part of MT population. The pause state can be introduced, for example, by the delay in depolymerization rate.

\section{REFERENCES}

[B17] A. BARlukova, Dynamic instability of microtubules and effect of microtubule targeting agents, PHD Thesis, Aix-Marseille University, France, March 2017.

[BGH+17] A. Barlukova, D. White, G. Henry, S. Honoré, F. Hubert Mathematical modeling of microtubule dynamic instability: new insight into the link between GTP-hydrolysis and microtubule aging, submitted 2017.

[DWJ95] WB. Derry, L. Wilson, and MA. Jordan, Substoichiometric binding of taxol suppresses microtubule dynamics, Biochemistry 34 (1995), no. 7, 2203-2211.

[HRT09] P. Hinow, V. ReZAnia, And JA. TuszyŃski, Continuous model for microtubule dynamics with catastrophe, rescue, and nucleation processes, Phys Rev E Stat Nonlin Soft Matter Phys 80 (2009), 031904.

[JW98] MA. JoRdAN AND L. Wilson, Microtubules and actin filaments: dynamic targets for cancer chemotherapy, Curr Biol 10 (1998), 123-130. 
$\left[\right.$ LBD $\left.^{+} 14\right]$ B. Lacroix, KG. Bourdages, JF. Dorn, S. Ihara, DR. Sherwood, PS. Maddox, and AS. Maddox, In situ imaging in c. elegans reveals developmental regulation of microtubule dynamics, Dev cell 29 (2014), 203-16.

$\left[\mathrm{MKD}^{+} 13\right]$ R. Mohana, EA. Katrukha, H. Doodhia, I. Smalb, E. Meijeringb, LC. Kapiteina, MO. SteinMEtzc, AND A. AKhmanova, End-binding proteins sensitize microtubules to the action of microtubule-targeting agents, PNAS 10 (2013), 8900-8905.

$\left[\right.$ PhM $\left.^{+} 12\right]$ A. Pagano, S. Honoré, R. Mohan, R. Berges, A. Akhmanova, And D. Braguer, Epothilone $b$ inhibits migration of glioblastoma cells by inducing microtubule catastrophes and affecting eb1 accumulation at microtubule plus ends, Biochem Pharmacol 84 (2012), no. 4, 432-43. 